\title{
Impact of Tube Voltage on Radiation Dose (CTDI) and Image Quality at Chest CT Examination
}

\author{
M. El Mansouri ${ }^{1^{\star}}$, A. Choukri ${ }^{1}$, M. Talbi ${ }^{2}$, O. K. Hakam ${ }^{1}$ \\ ${ }^{I}$ Ibn Tofail University, Faculty of Sciences, Department of Physics, Nuclear Physics and Techniques Team, \\ B.O 133, Kenitra, Morocco \\ ${ }^{2}$ Moulay Ismail University, Faculty of Sciences, Physical Sciences and Engineering, ENPT, \\ B.O. 11201, Zitoune Meknès, Morocco
}

\section{ARTICLE INFO}

\section{Article history:}

Received 27 Januari 2021

Received in revised form 16 March 2021 Accepted 16 March 2021

\section{Keywords:}

Chest examination

CTDI

Image quality

PMMA

Catphan 500

\begin{abstract}
A B S T R A C T
During Computed Tomography (CT) scan examinations, it is important to ensure a good diagnosis by providing the maximum information to detect pathologies and this can be done with a reduced dose. In this respect, several methods of dose reduction have been studied and evaluated. This work investigates the effect of tube voltage while varying the tube current on image quality and radiation dose at Chest CT examination. This study was conducted on HITACHI CT 16 slice Scanner using two phantoms for evaluating the dose and image quality; a PMMA phantom and a CATPHAN 500. Two tube voltages of $120 \mathrm{KVp}$ and $100 \mathrm{KVp}$ have been used for some variation of the tube currents (mAs) and recording the values of the measured quantities (CTDIv, spatial resolution, contrast to noise ratio CNR and noise). The scanning with $100 \mathrm{KVp}$ at Chest CT examination led to a reduction in CTDIv until $45 \%$, an increase of noise from $17 \%$ to $45 \%$, and the Spatial Resolution fell slightly ( 6 and $7 \mathrm{pl} / \mathrm{cm})$ compared to the $120 \mathrm{KVp}$. The CNR shows a slight regression from 11 to $22 \%$ for the $120 \mathrm{KVp}$ and $100 \mathrm{KVp}$. This study has shown that despite the increase in the image noise at low tube voltage $100 \mathrm{KVp}$, it is possible to reduce the radiation dose by up to $45 \%$ without degradation of image quality at Chest CT examination. Further works will evaluate the effect of acquisition parameters in other $\mathrm{CT}$ examinations.
\end{abstract}

(C) 2021 Atom Indonesia. All rights reserved

\section{INTRODUCTION}

Computed tomography (CT) scanning is a diagnostic imaging procedure that uses $\mathrm{x}$-rays to build cross-sectional images of the body. Several studies have been carried out on increasing of the CT scans undergoing on adult patients $[1,2]$. Nowadays, Morocco has recorded a significant technological evolution in the number of multi-slice CT scanners with more than 360 scanners and several radiology departments [3]. On one hand, these CT scanners have a high diagnostic capacity by reducing unidentified lesions- On the other hand, they provide high doses compared to other conventional radiology devices [4-6]. A single CT scan generates about 100 times or more radiation than a conventional $\mathrm{x}$-ray, or about one year of

${ }^{*}$ Corresponding author.

E-mail address: m.elmansouri@uhp.ac.ma

DOI: https://doi.org/10.17146/aij.2021.1120 radiation exposure from natural and artificial sources in the environment [7].

These exposures can lead to the development of radiation-induced cancers over time [8]. According to the ALARA principle, dose optimization is necessary to ensure optimal examination quality with a low dose [9-12]. Although the decrease in tube current (mAs) can reduce the dose, it can also confuse the diagnosis [13]. In this regard, several studies have been conducted to justify that it is possible to decrease mAs while reducing the dose without affecting image quality $[14,15]$.

It is important to ensure high-quality examinations with the lowest possible dose. These conditions depend on the choice of acquisition parameters. A reliable CT diagnosis requires highquality images with an optimal patient dose [15-18]. Despite the fact that Morocco has a very significant number of CT scanners, the quality assurance system following the recommendations of the IAEA 
and the CPIR has not been implemented [19]; Thus, the lack of prior research in this area prompted us to conduct this research, which aims to study the reduction of patient dose by assessing the influence of CT acquisition parameters that affect image quality.

Although decreasing $\mathrm{mAs}$ is the most effective way to reduce the CT radiation dose [20-22], this modification may also reduce image quality and affect diagnosis. Some studies have shown that scanning with a low tube voltage can reduce the dose without affecting image quality [23-25].

Therefore, this study aims to evaluate the effect of decreasing tube voltage $(\mathrm{KVp})$ while varying tube current (mAs), on image noise, contrast to noise ratio (CNR), spatial resolution (SR) and dose (CTDIv) at the Chest CT examination.

\section{MATERIALS AND METHODS}

The measurements have been performed on a HITACHI Supria scanner 16-slice, which has been installed in a Moroccan hospital since 2017 with a workload of about 30 patients per day. According to a questionnaire carried out at this hospital during 1 month, the tube voltage of $120 \mathrm{KVp}$ has been set at $85 \%$ of all thoracic examinations done.

In this study, two tube voltages of $120 \mathrm{KVp}$ and $100 \mathrm{KVp}$ have been used while varying the tube currents (mAs). These acquisition parameters are used for scanning through two phantoms evaluating the dose and image quality while recording the measured quantities' values (CTDIv, spatial resolution, contrast to noise ratio (CNR) and noise). These two phantoms were scanned three times for each acquisition parameter with tube currents from 75 to $300 \mathrm{mAs}$ to record a single average value.

The first phantom that evaluates the image quality is the type Catphan 500 (Phantom Laboratory, Salem NY, USA) (Fig 1(a)) [26]. It contains four modules. We have measured the CNR (module CTP515), the noise (module CTP486), and the spatial resolution (module CTP528) [27].

The image noise was the first measured quantity (Fig 1(c)). It is calculated using Eq. (1) [28].

$$
B=\frac{\sigma}{N S_{\text {eau }}-N S_{\text {air }}} \times 100
$$

where $\mathrm{B}$ (noise), $\sigma$ (standard deviation), $\mathrm{NS}_{\text {eau }}$ (number of water scanners), $\mathrm{NS}_{\text {air }}$ (number of air scanners).

The CNR was the second measured quantity using the image of the CTP515 module as shown in Eq. (2).

$$
C N R=\frac{\left(n b r C T_{\text {int }}-n b r C T_{\text {ext }}\right)}{S D}
$$

where $\mathrm{CT}_{\text {int }}$ is the $\mathrm{CT}$ number inside the circle, $\mathrm{CT}_{\text {ext }}$ is the CT number outside the circle, and SD is the standard deviation. CNR is calculated for the $15 \mathrm{~mm}$ circle, with a $1 \%$ contrast diameter of the CTP515 module's image [28].

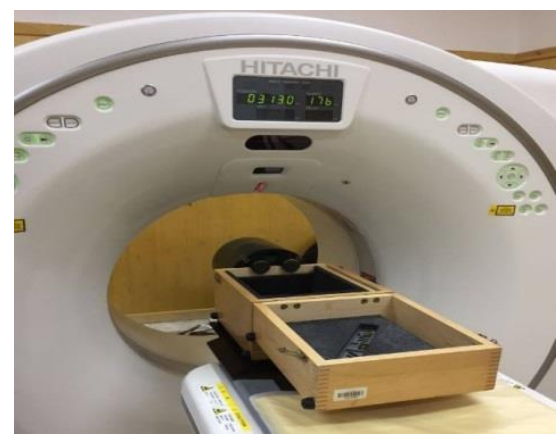

(a)

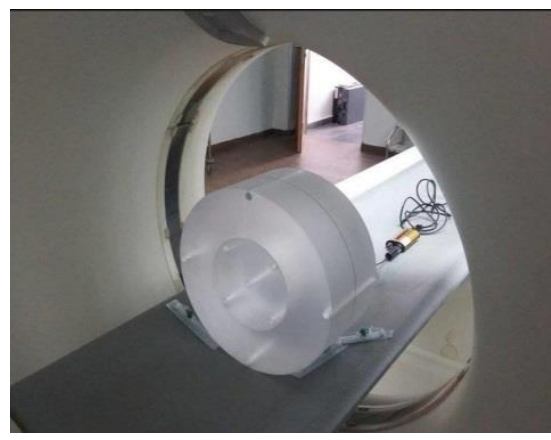

(b)

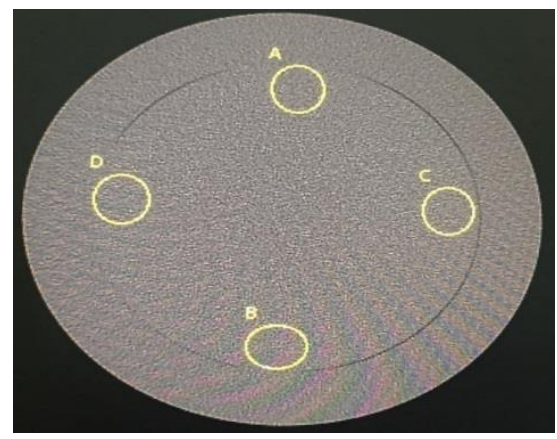

(c)

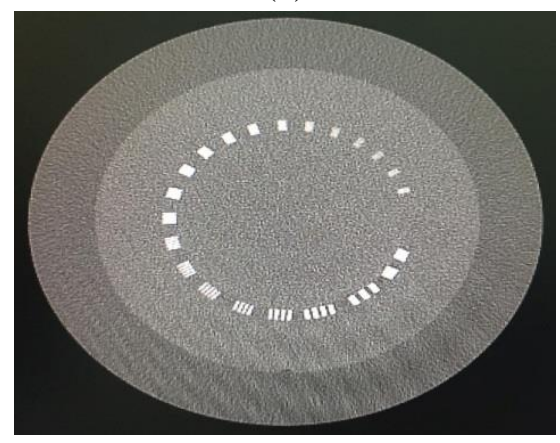

(d)

Fig. 1. (a) Catphan 500 for image quality assessment, (b) PMMA phantom for CTDI measurement of body with a calibrated pencil ionization chamber, (c) ROI's for image noise, (d) Spatial resolution. 
The Spatial Resolution (SR) was the last measured quantity (Fig. 1(d)) by counting the number of line pairs per centimeter [29].

The second phantom used to evaluate the dose is of PMMA (Polymethyl-Methacrylate) (Fig .1(b)), with $1.19 \mathrm{~g} / \mathrm{cm}^{3}$ density and contains five holes (center, 3, 6, 9, and 12 hour) [30]. A calibrated pencil ionization chamber (model 10X6-3CT) was also used [31], with an active length of $100 \mathrm{~mm}$, a measurement accuracy of $\pm 4 \%$, a dose measurement range of $200 \mathrm{nGy}-1 \mathrm{kGy}$, an electrometer type of RADCAL CORPORATION (California, USA), and an Accu-Gold+ interface software for displaying the output parameters.

The $\mathrm{CTDI}_{\mathrm{w}}$ was calculated according to Eq. (3).

$$
C T D I_{w}=\frac{1}{3} C T D I_{100, c}+\frac{2}{3} C T D I_{100, p}(\mathrm{mGy})
$$

where $\mathrm{CTDI}_{\mathrm{w}}$ is the weighted scanographic dose index; CTDI100, cat the center, and CTDI100, $\mathrm{p}$ at the periphery. The CTDIv was calculated by dividing the CTDIw by the factor of the pitch (1.06).

\section{RESULTS AND DISCUSSION}

This study focused on the measurement of CTDIv, a good CT index used to estimate radiation dose to patients. This index is a good indicator of comparison between the different acquisition parameters studied.

The CTDIv obtained at each variation of the acquisition parameters are presented in Table 1. The CTDIv reduction rates at $100 \mathrm{KVp}$ versus mAs were approximately $28-45 \%$ of those at $120 \mathrm{KVp}$. The CTDIv reduction rates at $100 \mathrm{KVp}$ compared to the CTDIv value at $120 \mathrm{KVp}$ and $166 \mathrm{mAs}$ were $63 \%$ at $75 \mathrm{mAs}, 59 \%$ at $90 \mathrm{mAs}$, $57 \%$ at $100 \mathrm{mAs}, 55 \%$ at mAs, $46 \%$ at $145 \mathrm{mAs}$, $43 \%$ at $166 \mathrm{mAs}, 29 \%$ at $200 \mathrm{mAs}, 23 \%$ at $250 \mathrm{mAs}, 6 \%$ at $300 \mathrm{mAs}$.

Table 1. CTDIv values obtained at each set of acquisition conditions.

\begin{tabular}{rrc}
\hline $\begin{array}{c}\text { Tube current } \\
\text { mAs }\end{array}$ & $\begin{array}{r}\text { CTDIv (mGy) } \\
\mathbf{1 2 0} \text { KVp }\end{array}$ & $\begin{array}{c}\text { CTDIv (mGy) } \\
\text { 100 KVp }\end{array}$ \\
\hline 75 & 5.82 & 3.88 \\
90 & 6.75 & 4.39 \\
100 & 7.70 & 4.64 \\
115 & 8.96 & 4.87 \\
145 & 9.86 & 5.81 \\
166 & 10.70 & 6.12 \\
200 & 11.50 & 7.66 \\
250 & 12.89 & 8.32 \\
300 & 14.09 & 10.16 \\
\hline
\end{tabular}

The measurements at each scan of the three image quality quantities, including noise, contrast to noise ratio and spatial resolution, are presented in Table 2.

Table 2. Noise, CNR and SR at $120 \mathrm{KVp}$ and $100 \mathrm{KVp}$.

\begin{tabular}{ccccccc}
\hline $\begin{array}{c}\text { Tube } \\
\text { current } \\
\text { mAs }\end{array}$ & \multicolumn{2}{c}{ Image noise } & \multicolumn{2}{c}{ CNR } & \multicolumn{2}{c}{ SR } \\
\cline { 2 - 7 } & $\mathbf{1 2 0}$ KVp & $\mathbf{1 0 0}$ KVp & $\mathbf{1 2 0}$ KVp & $\mathbf{1 0 0}$ KVp & $\mathbf{1 2 0}$ KVp & $\mathbf{1 0 0}$ KVp \\
\hline 75 & 8.20 & 9.86 & 0.76 & 0.60 & 6 & 6 \\
90 & 7.61 & 9.08 & 1.13 & 0.98 & 6 & 6 \\
100 & 7.36 & 8.62 & 1.33 & 1.12 & 6 & 6 \\
115 & 6.60 & 7.95 & 1.41 & 1.23 & 7 & 6 \\
145 & 5.02 & 7.12 & 1.56 & 1.34 & 7 & 6 \\
166 & 4.85 & 6.80 & 1.62 & 1.45 & 7 & 7 \\
200 & 4.46 & 6.25 & 1.70 & 1.60 & 7 & 7 \\
250 & 4.11 & 5.96 & 1.85 & 1.71 & 7 & 7 \\
300 & 3.69 & 5.12 & 1.98 & 1.80 & 8 & 7 \\
\hline
\end{tabular}

The spatial resolution (SR) was evaluated using the CTP528 module of the Catphan. The mean values were measured according to the variation of $\mathrm{mAs}$ at $120 \mathrm{KVp}$ and $100 \mathrm{KVp}$. For the $120 \mathrm{KVp}$, the values varied between 6 and $8 \mathrm{pl} / \mathrm{cm}, 6 \mathrm{pl} / \mathrm{cm}$ at $\mathrm{mAs}$ between (75-100), $7 \mathrm{pl} / \mathrm{cm}$ at $\mathrm{mAs}(115-250)$ and $8 \mathrm{pl} / \mathrm{cm}$ at $300 \mathrm{mAs}$. For the $100 \mathrm{KVp}$, the SR has been changed slightly between 6 and $7 \mathrm{pl} / \mathrm{cm}$.

The CNR was evaluated at the $15 \mathrm{~mm}$ diameter circle at a contrast of $1 \%$ of the CTP515 module image. It is found that the values of the $\mathrm{CNR}$ at $120 \mathrm{KVp}$ tube voltage increase with the increase in $\mathrm{mAs}$, from 0.76 to 1.98 . In addition, the rate of change between the parameters of $120 \mathrm{KVp}$ and $100 \mathrm{KVp}$ shows a slight regression from 11 to $22 \%$.

Figure 2 shows that the image noise was inversely correlated to the mAs. The lowest noise was observed at $120 \mathrm{KVp}-300 \mathrm{mAs}$, and the highest noise was observed at $100 \mathrm{KVp}-75 \mathrm{mAs}$. Compared to the noise obtained with $120 \mathrm{KVp}$ at $145 \mathrm{mAs}$, the noise obtained with $100 \mathrm{KVp}$ at 75-300 mAs was higher $(\mathrm{P}<0.001)$ which is consistent with the results of $\mathrm{C}$. Ludes et al [25].

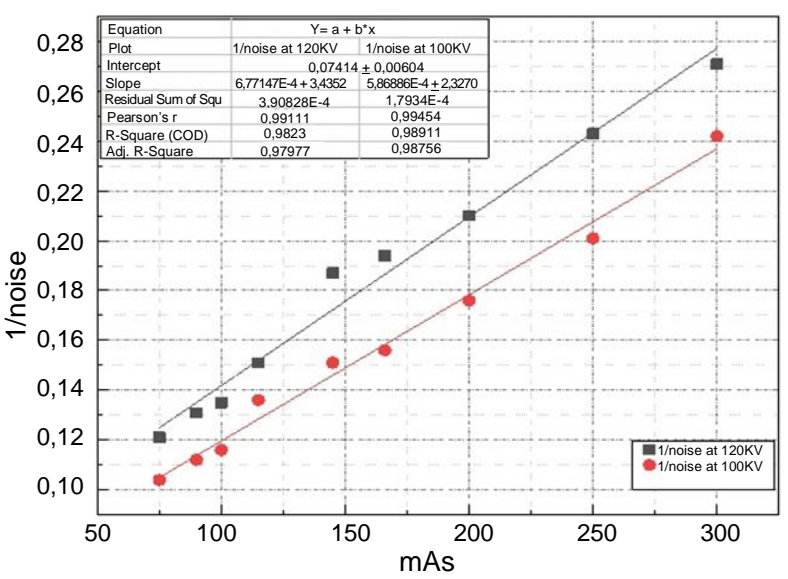

Fig. 2. Correlation between image noise and tube current mAs. 


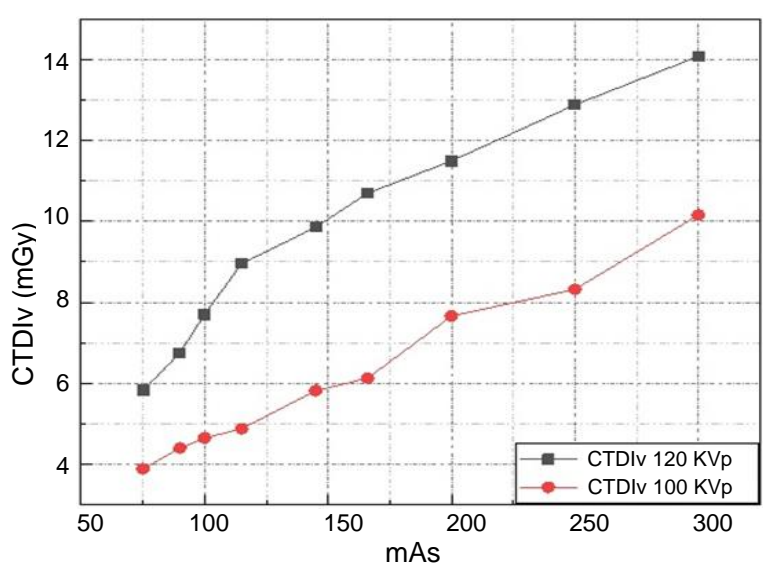

Fig. 3. CTDIv at $120 \mathrm{KVp}$ and $100 \mathrm{KVp}$.

In this study, we performed several different scans divided into two series, one for different $\mathrm{mAs}$ at $120 \mathrm{KVp}$ and the other at $100 \mathrm{KVp}$ while taking into account the image quality degradation.

Scanning with the tube voltage of $100 \mathrm{KVp}$ led to a reduction in CTDIv of 28 to $45 \%$ (Fig. 3) with an increase in noise of 17 to $45 \%$. This is consistent with the conclusions of Emilio Quaia [32], who concluded that the reduction in $\mathrm{KVp}$ allows the dose to be reduced without compromising image quality.

This study showed that scanning with a low tube voltage can reduce the dose without affecting image quality. This is consistent with the findings of Maria Taekker et al [33].

\section{CONCLUSION}

Several studies have investigated the reductions in parameters used to acquire CT protocols in several anatomical regions, such as the skull, chest, and abdomen. These studies have shown that reductions in the values of $\mathrm{mAs}$ and $\mathrm{KVp}$ made it possible to reduce the dose to patients, while preserving an adequate quality of images to establish a reliable diagnosis. The study results to the proposed protocol have shown that despite the increase in image noise at a low tube voltage, it is possible to reduce the radiation dose of patient (CTDIv) by up to $45 \%$ without causing a degradation of CNR and SR.

\section{ACKNOWLEDGMENT}

This research did not receive any specific grant from funding agencies in the public, commercial, or not-for-profit sectors.

\section{AUTHOR CONTRIBUTION}

M. El Mansouri, A. Choukri and M. Talbi equally contributed as the main contributors of this paper. O. K. Hakam contributed to analysis of data. All authors read and approved the final version of the paper.

\section{REFERENCES}

1. T. Koyama, Y. Zamami, A. Ohshima et al., Eur. J. Radiol. 97 (2017) 96.

2. C. M. Wright, M. K. Bulsara, R. Norman et al., Health Policy 121 (2017) 823.

3. H. Saikouk, I. Ou-Saada, F. Bentayeb et al., Med. Phys. Int. J. 7 (2019) 282.

4. C. Franck, P. Smeets, L. Lapeire et al., Phys. Medica 52 (2018) 32.

5. S. Semghouli, B. Amaoui, A. Kharras et al., J. Appl. Sci. Technol. 20 (2017) 1.

6. M. Alkhorayef, A. Sulieman, B. Alonazi et al., Radiat. Phys. Chem. 155 (2019) 65.

7. M. Mkimel, M. R. Mesradi, R. El Baydaoui et al., Perspect. Sci. 12 (2019) 100405.

8. P. Pinsky, D. S. Gierada, Lung Cancer 139 (2020) 179.

9. A. Serna, D. Ramos, E. Garcia-Angosto et al., Phys. Medica 55 (2018) 1.

10. K. Martini, J. W. Moon, M. P. Revel et al., Diagn. Interv. Imaging 101 (2020) 269.

11. J. B. Moser, S. L. Sheard, S. Edyvean et al., Clin. Radiol. 72 (2017) 407.

12. J. Amorim, B. Mendes, E. Ribau et al., Phys. Medica 32 (2016) 316.

13. J. Tugwell-Allsup, B. W. Owen and A. England, Radiogr. 27 (2021) 24.

14. J. Greffier, S. Boccalini, J. P. Beregi et al., Diagn. Interv. Imaging 101 (2020) 289.

15. B. Alikhani, L. Jamali, H. J. Raatschen et al., Radiogr. 23 (2017) 202.

16. M. K. Abdulkadir, N. A. Y. Mat Rahim, N. S. Mazlan et al., Radiat. Phys. Chem. 171 (2020) 108740.

17. K. P. Chang, T. K. Hsu, W. T. Lin et al., Radiat. Phys. Chem. 140 (2017) 260.

18. A. Manmadhachary, Y. Ravi Kumar and L. Krishnanand, Meas. 103 (2017) 18.

19. Anonymous, Quality Assurance Program for Computed Tomography: Diagnostic and Therapy Applications, Human Health Series No. 19, IAEA, Vienna (2012). 
20. B. R. Mussmann, S. D. Mørup, P. M. Skov et al., Radiogr. 27 (2021) 1.

21. F. Macri, J. Greffier, F. R. Pereira et al., Diagn. Interv. Imaging 97 (2016) 1131.

22. T. Kubo, Y. Ohno, M. Nishino et al., Eur. J. Radiol. Open 3 (2016) 86.

23. C. de Margerie-Mellon, C. de Bazelaire, C. Montlahuc et al., Acad. Radiol. 23 (2016) 1246.

24. Y. Ohno, H. Koyama, S. Seki et al., Eur. J. Radiol. 111 (2019) 93.

25. C. Ludes, A. Labani, F. Severac et al., Diagn. Interv. Imaging 100 (2019) 85.

26. Anonymous, Catphan ${ }^{\circledR} 500$ and 600 Manual, The Phantom Laboratory, https://www.uio.no/ studier/emner/matnat/fys/nedlagte-emner/ FYS4760/h07/Catphan500-600manual.pdf
27. M. J. Arsenault, M. J. Blanchette, M. F. Dinelle et al., Module de Contrôle de Qualité et de Radioprotection en Tomodensitométrie, CECR (2013).

28. A. M. A. Roa, H. K. Andersen and A. C. T. Martinsen, J. Appl. Clin. Med. Phys. 16 (2015) 350.

29. M. Rezaee and D. Letourneau, J. Med. Imaging Radiat. Sci. 50 (2019) 297.

30. Anonymous, PMMA Phantom for CT Performance with Convenience, Radcal ${ }^{\circledR}$ Corporation, No. 626 (2015) 91016.

31. Anonymous, The Chamber for Computed Tomography Dose Index (CTDI), Radcal ${ }^{\circledR}$ Corporation (2011) 4500054.

32. E. Quaia, Liver Pancreat. Sci. 1 (2016) 1.

33. M. Tækker, B. Kristjánsdóttir, O. Graumann et al, Clin. Imaging 74 (2021) 139. 